\title{
Fabric Defect Detection Using Local Homogeneity Analysis and Neural Network
}

\author{
Ali Rebhi, Issam Benmhammed, Sabeur Abid, and Farhat Fnaiech \\ Scientific Research Laboratory in Signal, Image Processing and Energy Mastery (SIME), University of Tunis, \\ 5 Avenue Taha Hussein, 1008 Tunis, Tunisia \\ Correspondence should be addressed to Ali Rebhi; ali_rebhi@yahoo.fr
}

Received 12 August 2014; Accepted 8 December 2014

Academic Editor: Ivan Moreno

Copyright (C) 2015 Ali Rebhi et al. This is an open access article distributed under the Creative Commons Attribution License, which permits unrestricted use, distribution, and reproduction in any medium, provided the original work is properly cited.

In the textile manufacturing industry, fabric defect detection becomes a necessary and essential step in quality control. The investment in this field is more than economical when reduction in labor cost and associated benefits are considered. Moreover, the development of a wholly automated inspection system requires efficient and robust algorithms. To overcome this problem, in this paper, we present a new fabric defect detection scheme which uses the local homogeneity and neural network. Its first step consists in computing a new homogeneity image denoted as $H$-image. The second step is devoted to the application of the discrete cosine transform (DCT) to the $H$-image and the extraction of different representative energy features of each DCT block. These energy features are used by the back-propagation neural network to judge the existence of fabric defect. Simulations on different fabric images and different defect aspects show that the proposed method achieves an average accuracy of $97.35 \%$.

\section{Introduction}

Defect detection is one of the main steps in quality control of manufacturing processes. In the fabric field, defect detection becomes an important task due to the widely used material in daily life. Fabric defects are responsible for nearly $85 \%$ of the defects found in the garment industry [1]. Also, it has been observed [2] that price of textile fabric is reduced by $45 \%$ to $65 \%$ due to defects. It is imperative, therefore, to detect, identify, and prevent these defects from reoccurring. A fabric defect is generally represented by a wired variation with respect to the overall surface aspect. Visual human inspection leads to usual errors due to several reasons, namely, the fatigue and fine defects. Hence, the automation of these operations helps to improve quality and reduce labor costs. As confirmed by $[3,4]$, there are more than 70 kinds of fabric defects defined by the textile industry. A human fabric inspection achieves a success rate about 60 to $75 \%$ [3]. Figure 1 shows the same machine automated inspection.

Automated visual inspection methods are increasingly taken into account in recent years. There are various visual inspection systems such as defect detection of tiles [5], woods $[6,7]$, ceramic [8], sheet steel [9], and textiles [10-19].
Fabric defect detection is one of the most scheming problems in visual inspection. Currently, there are a lot of new studies and researches in fabric inspection [10-19]. This paper emphasizes this problem and investigates some new technique to solve this problem.

1.1. Related Work. Texture analysis has a great interest in image processing, and there are many applications in its analyzing and processing, one of which is defect detection. Therefore, numerous methods are typically developed to solve the defect detection task in fabric images. The authors in [4] propose a review of the art field in this state. Chan and Pang [10] provided a comprehensive overview of fabric defect detection by Fourier analysis. Wavelet transform is another method which was applied in [11-14].

On the other hand, Latif-Amet et al. [15] proposed an approach that uses wavelet theory and cooccurrence matrix for the detection of defects encountered in textile images and classifies each subwindow as defective or nondefective by a Mahalanobis distance. Gabor filter has been widely used in the field of fabric defect detection. All the detection approaches using Gabor filter can be classified into two categories. One is to use a filter bank, such as $[16,17]$, and the 


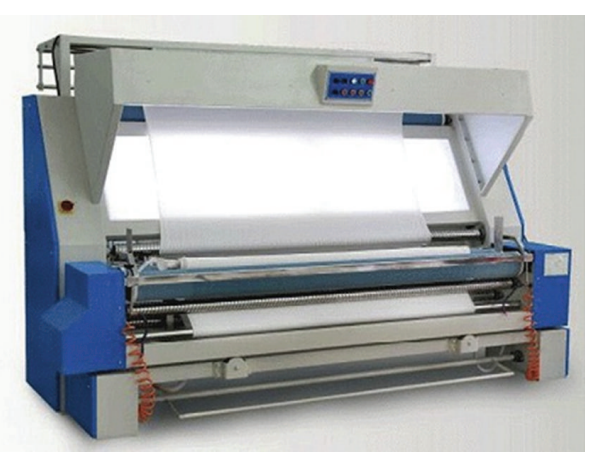

FIGURE 1: Machine automated inspection [4].

other one is to use optimal filters, such as [18-21]. In general, filtering with a filter bank can generate excessive data for processing though a set of filters may aid the segmentation. Correspondingly, the quality of localization and recognition is affected dramatically and the time consumption is large as well.

Moreover, model based approaches have been successfully used in the fabric defect detection. Some of textural analysis methods are based on the Markov random field model [22]. Campbell et al. [23] used the model based clustering method to detect linear pattern production defects.

Recently, neural network has attracted a lot of attention in defect detection applications. Huang and Chen [24] have used back-propagation neural network, with fuzzy logic, to achieve the classification of eight different kinds of fabric defects. In [25] an approach for the segmentation of local textile defects using feed-forward neural network is presented. Recently, Jing et al. in [26] present a machine vision system which uses basic patch statistics from raw image data combined with a two-layer neural network to detect surface defects on arbitrary textured and weakly labeled image data.

1.2. Present Work. In textured images, discontinuities represent generally an important feature such as boundaries. It is known that the local homogeneity analysis is the method to detect these discontinuities. Moreover, due to their nonparametric nature and their ability to describe complex decision regions, neural network is one of the best classifiers used for defect detection. In this paper, a new approach for fabric defect detection based on the local homogeneity analysis and neural network is proposed.

This paper is organized as follows. In Section 2 the local homogeneity computation ( $H$-image) for different window sizes is presented. The discrete cosine transform of the $H$ image and feature extraction are then described in Section 3. Section 4 provides a system description of the proposed method. It is followed by the experimental results in Section 5. Details of training and test procedures which include our proposed method for fabric defect detection and detection performance are described in Sections 5.1 and 5.2, respectively. Finally, Section 6 draws conclusion and summarizes the paper.

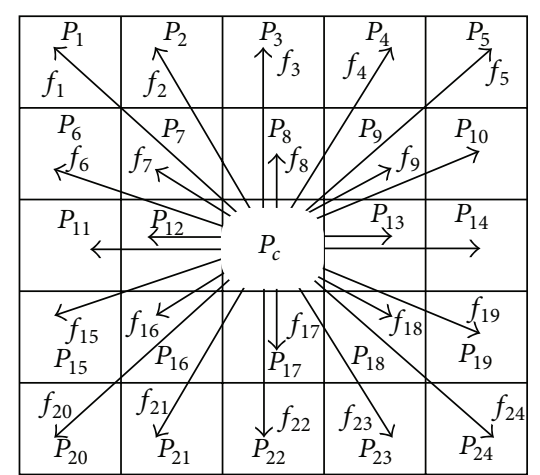

FIGURE 2: Different pixels used to compute the local homogeneity for $5 \times 5$ mask, for each pixel corresponds to a direction vector $f_{i}$.

\section{Local Homogeneity Definition}

It is known that discontinuities in textured images represent generally an important feature such as boundaries. The main problem is how to detect these discontinuities. A method to resolve this problem is the homogeneity $(H)$. The calculation of $H$ was proposed first by Jing et al. in [26]. Let $(x, y)$ be the coordinates of a pixel and $I(x, y)$ the corresponding intensity level. Let $P$ be a squared window, that is, a mask of $(2 N+1)$ width centered on the pixel. In the window $P$ one can define different vectors, such as $C p_{i}=\left(x_{i}-x_{c}, y_{i}-y_{c}\right)$, where $\left(x_{i}, y_{i}\right)$ is the neighbor of the center $\left(x_{c}, y_{c}\right)$ defined as

$$
\begin{aligned}
& c-N \leq x_{i} \leq c+N, \\
& c-N \leq y_{i} \leq c+N,
\end{aligned}
$$

where $\left(x_{i}, y_{i}\right)$ is the coordinate of the pixel $P_{i}, c$ is the coordinate of the centre pixel $\left(P_{c}\right)$, and $N$ is an integer number which defines the mask size.

Note that this definition allows computing the homogeneity of a pixel for different mask sizes $3 \times 3,5 \times 5$, and so forth.

Figure 2 shows an example of the different pixels used to compute homogeneity in a window of size $5 \times 5$ pixels.

Based on $C p_{i}$ a new vector $f_{i}$ is constructed as follows:

$$
f_{i}=\left(I\left(x_{i}, y_{i}\right)-I\left(x_{c}, y_{c}\right)\right) \frac{C p_{i}}{\left\|C p_{i}\right\|} .
$$

For a mask of width $(2 N+1)$ we have $(2 N+1)^{2}$ vectors $f_{i}$ : $1 \leq i \leq(2 N+1)^{2}$.

Let $f$ be the sum of the whole vectors defined in the window $P$, so

$$
f=\sum_{i=1}^{(2 N+1)^{2}} f_{i}
$$

The $H$ value measurement (the homogeneity value) is defined as the norm of $f$, so that

$$
H=\|f\|
$$


To limit the huge variation of the local homogeneity the normalized homogeneity is used:

$$
\widehat{H}=\frac{H-H_{\min }}{H_{\max }-H_{\min }},
$$

where $H_{\min }$ and $H_{\max }$ are the minimum and the maximum value of homogeneity, respectively. The resulting $H$-image is constructed by the different values of $\widehat{H}$ computed for each pixel value $I_{c}\left(x_{c}, y_{c}\right)$.

\section{Discrete Cosine Transform (DCT) and Features Extraction from the DCT Block}

3.1. Brief Description of DCT Transform. The DCT of an $H$ image $H_{x, y}$ of size $M \times N$ is given by (6). This expression must be computed for values of $u=0,1,2, \ldots, M-1$ and also for $v=0,1,2, \ldots, N-1$.

The variables $u$ and $v$ are frequency variables, $x$ and $y$ are spatial variables, and $P$ and $Q$ are the size of the DCT block:

$D_{u, v}$

$$
=\sigma(u) \sigma(v) \sum_{x=0}^{P-1} \sum_{y=0}^{Q-1} H_{x, y} \cos \left(\frac{(2 y+1) u \pi}{2 P}\right) \cos \left(\frac{(2 x+1) v \pi}{2 Q}\right),
$$

where

$$
\begin{gathered}
\sigma(u)= \begin{cases}\sqrt{\frac{1}{P}}, & \text { for } u=0 \\
\sqrt{\frac{2}{P}}, & \text { otherwise, }\end{cases} \\
\sigma(v)= \begin{cases}\sqrt{\frac{1}{Q}}, & \text { for } v=0 \\
\sqrt{\frac{2}{Q}}, & \text { otherwise. }\end{cases}
\end{gathered}
$$

3.2. Feature Extraction. In order to characterize the frequency domain within texture image, this paper proposes to use the features energy distribution in the 2D-DCT domain from the $H$-image, which is presented as follows [27]:

(i) The horizontal energy value of the DCT block:

$$
E_{H}=\sum_{u=0}^{P-1} \sum_{v=0}^{Q-1}\left[(v+1)^{2} \times H_{x, y}^{2}\right]^{1 / 2} \quad \text { for } u+v \neq 0 .
$$

(ii) The vertical energy value of a DCT block:

$$
E_{V}=\sum_{u=0}^{P-1} \sum_{v=0}^{Q-1}\left[(u+1)^{2} \times H_{x, y}^{2}\right]^{1 / 2} \quad \text { for } u+v \neq 0 .
$$

(iii) The diagonal energy value of a DCT block:

$$
E_{D}=\sum_{u=0}^{P-1} \sum_{v=0}^{Q-1}\left[(u+1) \times(v+1) \times H_{x, y}^{2}\right]^{1 / 2} \quad \text { for } u+v \neq 0 .
$$

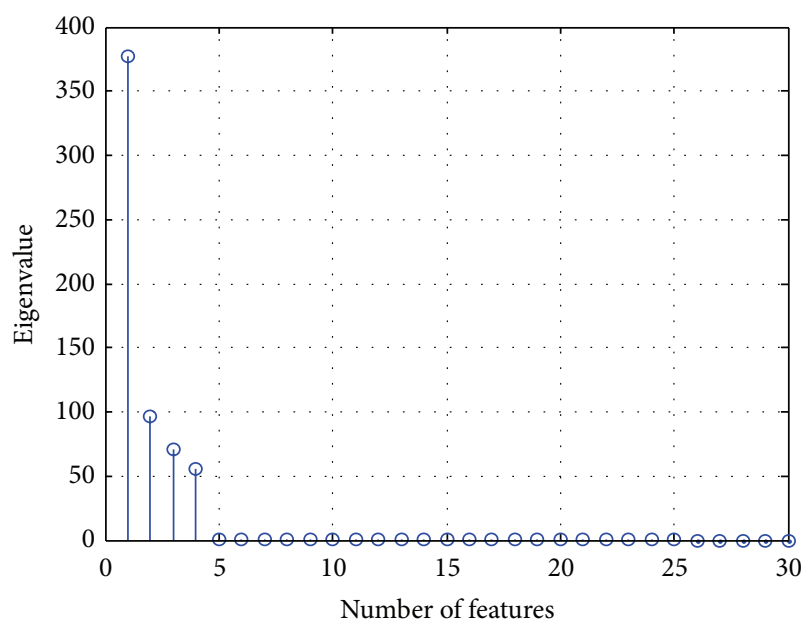

FIGURE 3: Eigenvalues of covariance matrix for feature reduction.

(iv) The average energy value of a DCT block:

$$
E_{M}=\frac{1}{P \times Q} \sum_{u=0}^{P-1} \sum_{v=0}^{Q-1} H_{x, y}^{2}
$$

(v) The standard deviation energy value of a DCT block:

$$
E_{S}=\frac{1}{P \times Q-1} \sum_{u=0}^{P-1} \sum_{v=0}^{Q-1}\left(H_{x, y}-E_{M}\right)^{2}
$$

Then the features vector of each pixel is expressed as

$$
T=\left[\begin{array}{lllll}
E_{H} & E_{V} & E_{D} & E_{M} & E_{S}
\end{array}\right] .
$$

In the next section, the principal component analysis (PCA) is introduced to eliminate correlations between features and reduce the dimensionality of the original feature vectors.

3.3. Features Reduction. Feature reduction means transforming the original features into a lower dimensional space [28, 29]. Most of the feature extraction techniques have been based on linear techniques such as principal component analysis (PCA). PCA is a quantitatively rigorous method for achieving data dimensionality reduction. The method generates a new set of variables, called principal components (PCs), which maximize the variance of the projected vectors. Each PC is a linear combination of the original variables. All the PCs are orthogonal to each other, so there is no redundant information. The PCs as a whole form an orthogonal basis for the space of the data. Thus, the first PC consists of the highest variability, the second PC consists of the next highest variability, and so on for other directions. The first few components are kept and others with less variability are discarded. As shown in Figure 3, in the proposed work, the first 4 PCs are used to feed to the feed-forward neural networks (FFN) classifier. 

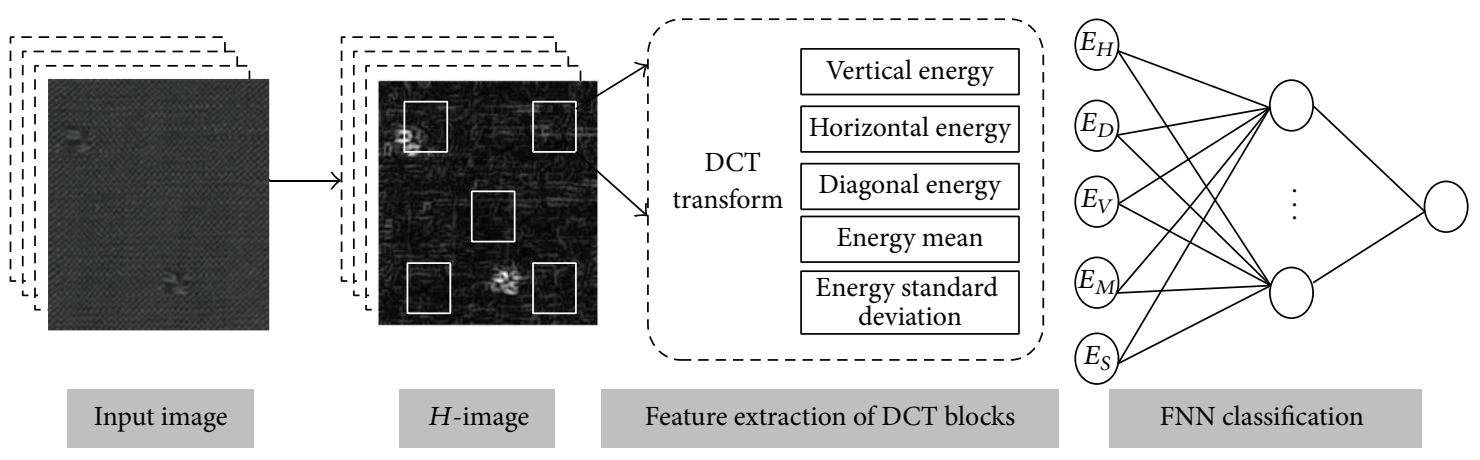

FIGURE 4: Block diagram of fabric defect detection using $H$-image and FFN.

\section{Fabric Defect Detection Method}

4.1. Fabric Defect Classification Based on FFN. This method consists in scanning the acquired image with a squared window and computing the local homogeneity for each pixel and constructing the $H$-image. In our work we have used a window of size $11 \times 11$ pixels. Then, we divided the $H$-image into overlapping squared blocks. The five aforementioned types of energy are computed through the DCT applied for each block which produces these energies. Every pixel of the acquired image is characterized by a feature vector (five types of energy). Then, the statistical or $Z$-score normalization technique is applied to all the features vectors that form the input to the neural network. The number of the inputs of the neural network is not equal to the same number of feature extractions. They are obtained using a principal component analyzing (PCA) routine. This routine is applied in order to limit the number of inputs of neural network. In this case the number of inputs equals four (i.e., the number of chosen principal components). The number of the training vectors equals the number of pixels in the considered field of view of the investigated fabric surface. The network has a single output.

The concept diagram of the fabric defect detection scheme is presented in Figure 4.

The procedure of the proposed method can be summarized as follows.

Step 1. Scanning of the input image $I$ with a squared window and computing the local homogeneity for each pixel.

Step 2. Normalization of the local homogeneity.

Step 3. Division of the $H$-image into overlapping squared blocks and application of a DCT transform.

Step 4. Features extraction from the DCT block.

Step 5. Features reduction using the PCA method.

Step 6. Normalization of all features using Zero mean and unity variance technique.

Step 7. Classification process for fabric defect by FFN based on $H$-image (since only 2 conditions (block contains defect regions or not) need to be identified in this paper).
4.2. Learning Defect Classifier. After a feature calculation step, now we use the training data to train a neural network using back-propagation. An example of a FFN structure is shown in Figure 4 FFN classification. The size of the input layer is equal to the feature dimension; here $k=4$ (after applying a PCA technique the number of features is reduced to 4). The second layer, called the hidden layer, has $L 1$ nodes. In the output layer, all nodes from the hidden layer are mapped to one final node showing the classification result. All weights in the neural network are initialized by random numbers. Training a network means optimizing network detection performance. This is realized by minimizing the performance function. The performance measurement function in this case is the mean squared error (MSE). The final classification result is a value between $[-1,1]$ and predicts whether an unknown block contains defect regions or not.

\section{Experimental Results: Application of the Proposed Method in Fabric Defect}

This section presents the experimental results for fabric defect detection. The dataset used is established and the results are analyzed in Section 5.1. The performance of the proposed algorithm for a number of sample images is presented and analyzed in Section 5.2. Discussion and comparison with some previous works are given in Section 5.3.

5.1. Dataset. The dataset used in this work is provided by PARTNER textile industry in Tunisia [30]. The dataset consists of 89 images, of which 13 have no defects and 76 have various fabric defects. The total databases were divided into two sets: one for training (containing $60 \%$ of the samples) and the other for test (containing $40 \%$ of the samples). Table 1 summarizes some fabric defects types used in this study, their definition, and their reasons.

All simulations were implemented on a personal computer (Intel core 2Duo CPU T5870 @2 GHz and 3GB of RAM). The programs were tested first in MATLAB language and after that they were rewritten in $\mathrm{C}++$ language for the purpose to be implemented in a real time process. We note that once the neural network is trained in the learning phase (offline step) it will be inserted in the defect detection process and then the global needed time is considerably reduced. 
TABLE 1: Example of fabric defect types.

\begin{tabular}{|c|c|c|}
\hline Defect type & Definition & Reasons \\
\hline Floats & $\begin{array}{l}\text { A portion of a yarn in a fabric that extends or floats, } \\
\text { unbound, over two or more adjacent ends or picks }\end{array}$ & $\begin{array}{l}\text { It is caused by missing of interlacement of two series of } \\
\text { threads }\end{array}$ \\
\hline Holes & A fabric area free of both warp and weft threads & It is a mechanical fault caused by a broken machine part \\
\hline Oil stains & Fabric area contains oil spots & $\begin{array}{l}\text { It is caused by too much oiling on loom parts or from } \\
\text { other external sources }\end{array}$ \\
\hline Slubs & A local uneven fabric thickness & $\begin{array}{l}\text { It is caused by an extra piece of yarn that is woven into } \\
\text { fabric. It can also be caused by thick places in the yarn } \\
\text { or by fly waste being spun in yarn during the spinning } \\
\text { process }\end{array}$ \\
\hline Knots & $\begin{array}{l}\text { A fabric place where two ends of yarn have been tied } \\
\text { together and the tails of the knot are protruding from } \\
\text { the surface }\end{array}$ & It is caused by tying spools of yarn ends together \\
\hline Miss-end & $\begin{array}{l}\text { A warp thread is absent in the fabric for a short or long } \\
\text { distance }\end{array}$ & $\begin{array}{l}\text { It is due to incorrect warping or by a broken warp } \\
\text { thread that never replaced by another one }\end{array}$ \\
\hline Miss-pick & $\begin{array}{l}\text { A weft thread is absent in the fabric for a short or long } \\
\text { distance }\end{array}$ & $\begin{array}{l}\text { It is caused by incorrect picking or if the weaver } \\
\text { restarted the loom after any stoppage without adapting } \\
\text { the position for the new insertion }\end{array}$ \\
\hline
\end{tabular}

TABLE 2: Training parameters.

\begin{tabular}{lc}
\hline Parameter & Value \\
\hline Number of inputs/outputs & $4 / 1$ \\
Number of hidden layers & 1 \\
Number of hidden layer neurons & 9 \\
Learning coefficient for hidden layer & 0.6 \\
Activation function & Bipolar Sigmoid \\
Initial weight values (random) & $0 \sim 0.1$ \\
Learning rule delta & Delta \\
Convergence error & $2.8 e-5$ \\
Number of iterations & 50120 \\
\hline
\end{tabular}

Training of the network was carried out with the error back-propagation algorithm by using a set of fabric images data containing 46 images with defect and 8 images without defect covering every possible pattern of defect blocks and nondefect blocks.

Initially, all the weight values of the neural network were randomly set to small values between 0 and 0.1 and the learning coefficients were set to 0.6. Several experiments were conducted for selecting the best FFN. The factors that played a significant role in the performance of the system were the number of hidden layer neurons, the learning coefficients of the hidden layer neurons and the output layer, the activation function, and the learning rule. The parameters of the best architecture for FFN are summarized in Table 2.

5.2. Performance Detection. After the FFN is trained with 54 images, its performance has been tested with the remaining ones (35 images). The performance of this algorithm is validated by calculating the following performance measure for the train set and test set separately. The classification accuracy (CA) is as follows: ratio between the total number
TABle 3: Definitions of true positive (TP), false positive (FP), true negative (TN), and false negative (FN) in defect detection.

\begin{tabular}{lcc}
\hline & Actually defective & Actually defect-free \\
\hline Detected as defective & True positive (TP) & False positive (FP) \\
\hline Detected as defect-free & False negative (FN) & True negative (TN) \\
\hline
\end{tabular}

of correctly classified test samples and the total number of test samples. Generally, detection accuracy, also known as detection success rate, is defined as

$$
\mathrm{CA}[\%]=\frac{\mathrm{TP}+\mathrm{TN}}{\mathrm{TP}+\mathrm{FN}+\mathrm{TN}+\mathrm{FP}} \times 100 .
$$

Table 3 outlines definitions of true positive (TP), false positive (FP), true negative (TN), and false negative (FN) in defect detection.

The classification results of all classifiers in the training and testing processes are presented in Table 4. In the training process, all the FFN classifiers achieve an average accuracy of $98.30 \%$ and $99.05 \%$ in the whole dataset and the reduced dataset, respectively. This indicates that the classifiers are well trained and can be applied for fabric defect detection. However, in the testing process these classifiers are validated against the test data; the average accuracy is about $97.35 \%$ and $96.25 \%$ for the reduced and original features, respectively.

From the test results shown in Table 4 it can be seen that the FNN classifiers recognize the blocks defects effectively. The recognition results of FNN are ideal because of its high accuracy and a good generalization capability, when the average classification efficiency close to $97.35 \%$ is reasonably good.

For $256 \times 256$ image the time needed for defect detection by scanning all the image is about 0.8 seconds. Taking into account the production rate (knitting speed), the algorithm can be used in a real time application. 
TABLE 4: The testing accuracy for different blocks conditions using FNN.

\begin{tabular}{|c|c|c|c|c|}
\hline \multirow{3}{*}{ Blocks conditions } & \multicolumn{2}{|c|}{ FNN including all features } & \multicolumn{2}{|c|}{ FNN including selected features } \\
\hline & \multicolumn{2}{|c|}{ Classification accuracy [\%] } & \multicolumn{2}{|c|}{ Classification accuracy [\%] } \\
\hline & Training & Testing & Training & Testing \\
\hline Defective blocks & 97.50 & 95.30 & 98.30 & 96.60 \\
\hline Nondefective blocks & 99.10 & 97.20 & 100 & 98.10 \\
\hline Average & 98.30 & 96.25 & 99.15 & 97.35 \\
\hline
\end{tabular}

TABLE 5: Comparison of the proposed method with some previous research.

\begin{tabular}{|c|c|c|c|}
\hline Literature & Features & Classifier & Accuracy [\%] \\
\hline [29] & $\begin{array}{l}\text { Complex symmetric Gabor filter bank and principal } \\
\text { component analysis (PCA) }\end{array}$ & PCA + Euclidean norm & 98.8 \\
\hline$[31]$ & $\begin{array}{l}\text { Wavelet based feature extraction and morphological } \\
\text { operations + Dempster-Shafer theory }\end{array}$ & MLP neural networks & 89.48 \\
\hline$[32]$ & $\begin{array}{l}\text { Radial basis function (RBF) network and gray level } \\
\text { arrangement in the neighborhood of each pixel + PCA }\end{array}$ & PCA + RBF network with Gaussian kernel & 83.4 \\
\hline$[33]$ & Small scale overcomplete basis set and Gabor filter & Sparse coding & 93 \\
\hline $\begin{array}{l}\text { Proposed } \\
\text { Methodology }\end{array}$ & $\begin{array}{l}\text { Features derived from DCT transform of } H \text {-image and } \\
\text { FFN }\end{array}$ & $\mathrm{PCA}+\mathrm{FFN}$ & 97.35 \\
\hline
\end{tabular}

5.3. Discussion and Comparison with Some Previous Works. In this experiment, the $H$-image is obtained after scanning the input image using a window of size $11 \times 11$ pixels. The features extractions are produced by dividing the $H$-image into overlapping blocks of size $4 \times 4$ pixels after applying the DCT transform. The final classification result is obtained from a feed-forward network trained with the back-propagation (BP). Figure 5 shows detection examples of the fabric dataset. The kinds of fabric defects vary in size, shape, and orientation.

In Figure 5, column (a) shows the original images. Columns (b) and (c), respectively, correspond to the $H$-image and defect detection results. Image in the first line represents the "hole" defect. The proposed algorithm was able to correctly detect the defect. Image in the second line shows another type of defect (slub defect) and the corresponding defects detection results which were accurately detected. Other types of sample defects are shown in the rest of the figure.

It is interesting to note that despite the varying size and orientation of the defects, the algorithm managed to identify accurately most of the defects.

Based on these results, it can be concluded that the DCT features extraction method effectively improves classification performance for the given fabric defect classification problem.

Table 5 provides a summary of the studies on automated fabric defect detection. Bissi et al. (2013) [29] proposed an approach for automated texture defect detection in uniform and structured fabrics based on a complex symmetric Gabor filter bank and PCA. The performance of their algorithm has been extensively evaluated by using images of the TILDA Textile Texture Database and reported an accuracy of $98.8 \%$. Tabassian et al. (2011) [31] used a wavelet based feature extraction method beside binary thresholding technique and morphological operations and classified them by MLP neural networks. The Dempster-Shafer theory of evidence was then employed for combining different evidence to decrease the value of uncertainty. It achieved a detection accuracy of 89.48\%. Zhang et al. (2010) [32] used the neighborhood of each pixel to extract the features and to reduce them using PCA and classified them using radial basis function (RBF) network improved by Gaussian mixture model (GMM) with an accuracy of $83.4 \%$. Qiuping et al. (2014) [33] used an approach for defect detection applied to twill, plain, gingham, and striped fabric using Gabor filter and sparse coding with an accuracy of $93 \%$.

In conclusion, the evaluation of our method versus those in previous researches, given in Table 5, shows that our experiment has good potential for improving fabric defect detection. The features derived from the DCT transform of $H$-image with the FFN classifier produce a good classification performance: the average CA result is $97.35 \%$. This combination of the feature and classifier is promising for a high accuracy of fabric defect detection.

\section{Conclusions and Future Work}

In this paper a novel method is proposed to solve the problem of fabric defect detection, which is based on the local homogeneity analysis and the feed-forward neural networks. A DCT transform based feature extraction method beside the PCA was used. The performance of this algorithm has been extensively evaluated by using images of the PARTNER Textile Texture Database. The comparison with other existing algorithms reported in literature highlights the effectiveness of the proposed approach. The average accuracy of block condition was $97.35 \%$; the results show that the proposed algorithm is a strong technique for fabric defect detection. 

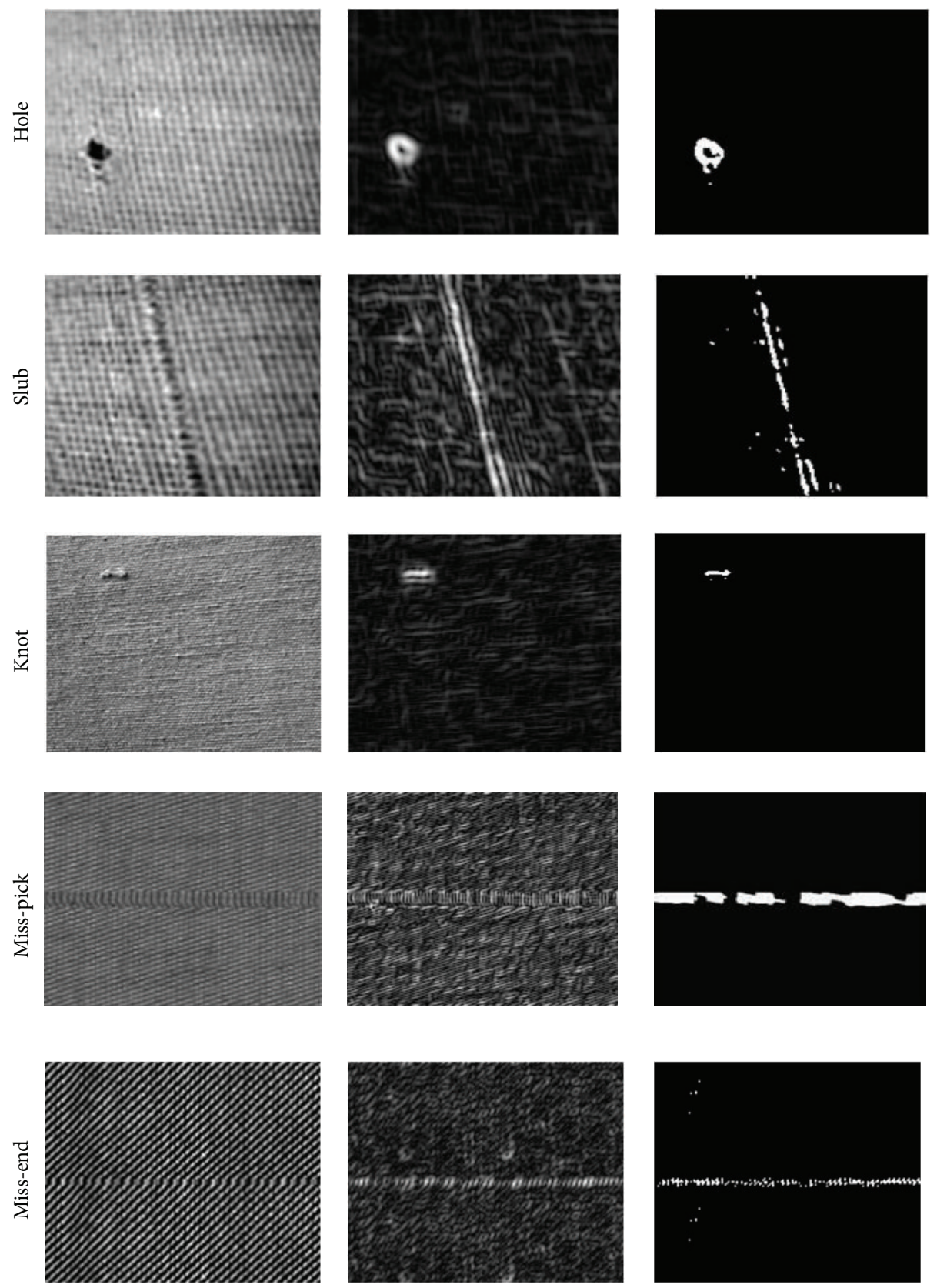

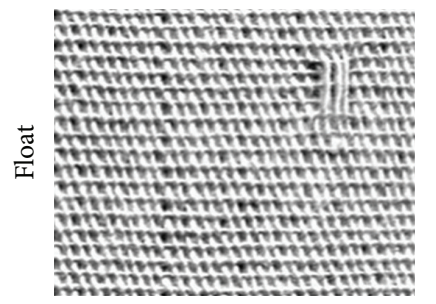

(a)

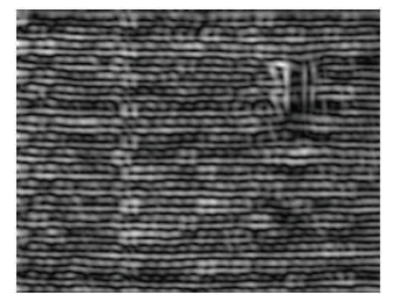

(b)

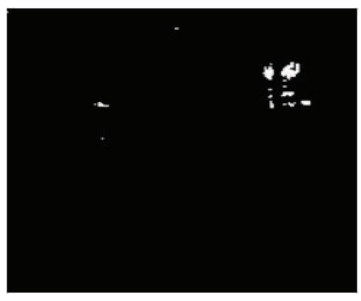

(c)

FiguRE 5: Defect detection results. (a) Images of fabrics with different types of defects, (b) the corresponding $H$-image, and (c) the corresponding defect detection results. 
The algorithm presents some drawbacks, namely, the arbitrarily choice of the windows size of the mask used to compute the $H$-image. This in its turn affects intensively the defect detection of the algorithm. Indeed if the mask size is chosen relatively large (such as $15 \times 15$ ) this will homogenize even some different regions and then this will cause some false alarms. If the mask size is chosen relatively small (such as $3 \times 3$ or $5 \times 5$ ) this will induce a poor homogenization and, therefore, similar areas (especially in stochastic textures) will be considered as different areas and may be seen as defects by the algorithm.

\section{Conflict of Interests}

The authors declare that there is no conflict of interests regarding the publication of this paper.

\section{Acknowledgment}

The authors would like to thank Mr. Mohamed Ali Derbel Director of the PARTENER Textile for helpful discussion and for providing the fabric image database.

\section{References}

[1] P. Sengottuvelan, A. Wahi, and A. Shanmugam, "Automatic fault analysis of textile fabric using imaging systems," Research Journal of Applied Sciences, vol. 3, pp. 26-31, 2008.

[2] T. Vikrant and S. Gaurav, "Automatic fabric fault detection using morphological operations on bit plane," International Journal of Engineering Research \& Technology, vol. 2, pp. 856-861, 2013.

[3] Hong Kong Productivity Council, Textile Handbook 2000, The Hong Kong Cotton Spinners Association, 2000.

[4] H. Y. T. Ngan, G. K. H. Pang, and N. H. C. Yung, "Automated fabric defect detection-a review," Image and Vision Computing, vol. 29, no. 7, pp. 442-458, 2011.

[5] M. Ghazvini, S. A. Monadjemi, N. Movahhedinia, and K. Jamshidi, "Defect detection of tiles using 2D-wavelet transform and statistical features," World Academy of Science, Engineering and Technology, vol. 37, pp. 901-904, 2009.

[6] R. W. Conners, C. W. McMillin, and K. Lin, "Identifying and locating surface defects in wood: part of an automated lumber processing system," IEEE Transactions on Pattern Analysis and Machine Intelligence, vol. 5, no. 6, pp. 573-583, 1983.

[7] D. Zhu, R. Conners, and P. Araman, "CT image sequence processing for wood defect recognition," in Proceedings of the IEEE 23rd Southeastern Symposium on System Theory, pp. 7579, Columbia, SC, USA, 1991.

[8] C. Boukouvalas, "Color grading of randomly textured ceramic tiles using color histograms," IEEE Transactions on Industrial Electronics, vol. 46, no. 1, pp. 219-226, 1999.

[9] K. Wiltschi, A. Pinz, and T. Lindeberg, "Automatic assessment scheme for steel quality inspection," Machine Vision and Applications, vol. 12, no. 3, pp. 113-128, 2000.

[10] C.-H. Chan and G. K. H. Pang, "Fabric defect detection by Fourier analysis," IEEE Transactions on Industry Applications, vol. 36, no. 5, pp. 1267-1276, 2000.

[11] X. Yang, G. Pang, and N. Yung, "Robust fabric defect detection and classification using multiple adaptive wavelets," IEE
Proceedings_Vision, Image and Signal Processing, vol. 152, pp. 715-723, 2005.

[12] D. M. Tsai and B. Hsiao, "Automatic surface inspection using wavelet reconstruction," Pattern Recognition, vol. 34, no. 6, pp. 1285-1305, 2001.

[13] Y. X. Zhi, G. K. H. Pang, and N. H. C. Yung, "Fabric defect detection using adaptive wavelet," in Proceedings of the IEEE Interntional Conference on Acoustics, Speech, and Signal Processing (ICASSP '01), pp. 3697-3700, May 2001.

[14] Y. Han and P. Shi, "An adaptive level-selecting wavelet transform for texture defect detection," Image and Vision Computing, vol. 25, no. 8, pp. 1239-1248, 2007.

[15] A. Latif-Amet, A. Ertüzün, and A. Erçil, "An efficient method for texture defect detection: sub-band domain co-occurrence matrices," Image and Vision Computing, vol. 18, no. 6, pp. 543$553,2000$.

[16] A. Kumar and G. K. H. Pang, "Fabric defect segmentation using multichannel blob detectors," Optical Engineering, vol. 39, no. 12, pp. 3176-3190, 2000.

[17] A. Kumar and G. K. H. Pang, "Defect detection in textured materials using Gabor filters," IEEE Transactions on Industry Applications, vol. 38, no. 2, pp. 425-440, 2002.

[18] A. Hamid, A. Alireza, and S. Esmaeil, "Defect detection in textiles using morphological analysis of optimal Gabor wavelet," in Proceedings of the International Conference on Computer and Automation Engineering (ICCAE '09), pp. 26-30, 2009.

[19] A. Bodnarova, M. Bennamoun, and S. Latham, "Optimal gabor filters for textile flaw detection," Pattern Recognition, vol. 35, no. 12, pp. 2973-2991, 2002.

[20] R. Han and L. M. Zhang, "Fabric defect detection method based on Gabor filter mask," in Proceedings of the WRI Global Congress on Intelligent Systems (GCIS '09), pp. 184-188, Xiamen, China, May 2009.

[21] M. Li and R. C. Staunton, "Optimum Gabor filter design and local binary patterns for texture segmentation," Pattern Recognition Letters, vol. 29, no. 5, pp. 664-672, 2008.

[22] S. Ozdemir and A. Ercil, "Markov random fields and Karhumen-Loeve transforms for defect inspection of textile products," in Proceedings of the IEEE Conference on Emerging Technologies and Factory Automation, vol. 2, pp. 697-703, 1996.

[23] J. G. Campbell, C. Fraley, F. Murtagh, and A. E. Raftery, "Linear flaw detection in woven textiles using model-based clustering," Pattern Recognition Letters, vol. 18, no. 14, pp. 1539-1548, 1997.

[24] C.-C. Huang and I.-C. Chen, "Neural-fuzzy classification for fabric defects," Textile Research Journal, vol. 71, no. 3, pp. 220224, 2001.

[25] A. Kumar, "Neural network based detection of local textile defects," Pattern Recognition, vol. 36, no. 7, pp. 1645-1659, 2003.

[26] F. Jing, M. Li, H.-J. Zhang, and B. Zhang, "Unsupervised image segmentation using local homogeneity analysis," in Proceedings of the IEEE International Symposium on Circuits and Systems (ISCAS '03), pp. II456-II459, IEEE, May 2003.

[27] Y.-S. Chiu and H.-D. Lin, "An innovative blemish detection system for curved LED lenses," Expert Systems with Applications, vol. 40, no. 2, pp. 471-479, 2013.

[28] S. Ding, C. Li, and Z. Liu, "Fabric defect detection scheme based on Gabor filter and PCA," Advanced Materials Research, vol. 482-484, pp. 159-163, 2012.

[29] L. Bissi, G. Baruffa, P. Placidi, E. Ricci, A. Scorzoni, and P. Valigi, "Automated defect detection in uniform and structured fabrics using Gabor filters and PCA," Journal of Visual Communication and Image Representation, vol. 24, no. 7, pp. 838-845, 2013. 
[30] 2013, http://www.partnertextile.com/.

[31] M. Tabassian, R. Ghaderi, and R. Ebrahimpour, "Knitted fabric defect classification for uncertain labels based on DempsterShafer theory of evidence," Expert Systems with Applications, vol. 38, no. 5, pp. 5259-5267, 2011.

[32] Y. Zhang, Z. Lu, and J. Li, "Fabric defect classification using radial basis function network," Pattern Recognition Letters, vol. 31, no. 13, pp. 2033-2042, 2010.

[33] Z. Qiuping, W. Minyuan, L. Jie, and D. Dexiang, "Fabric defect detection via small scale over-complete basis set," Textile Research Journal, vol. 84, no. 15, pp. 1634-1649, 2014. 

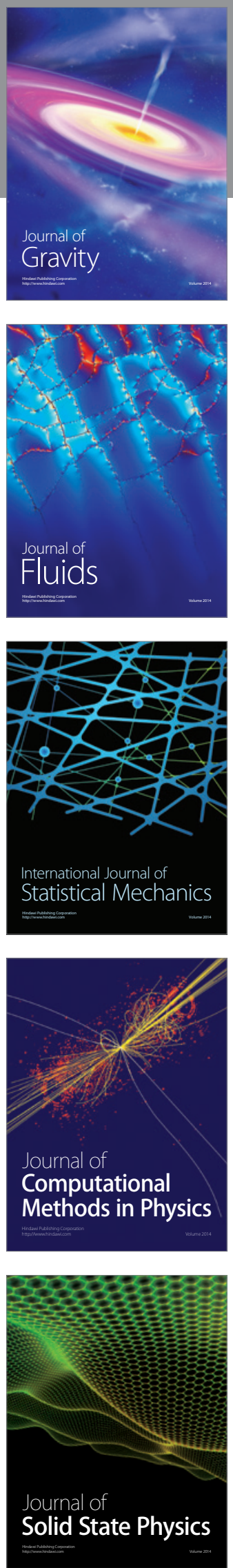

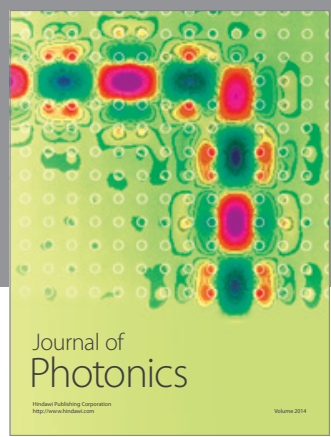

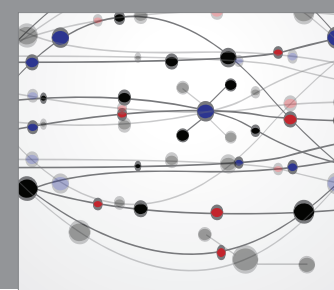

The Scientific World Journal

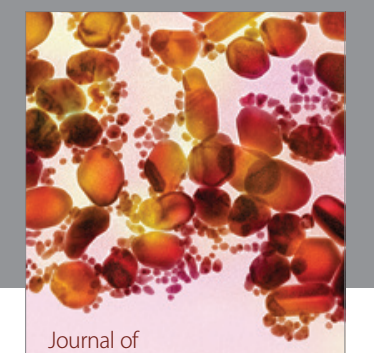

Soft Matter
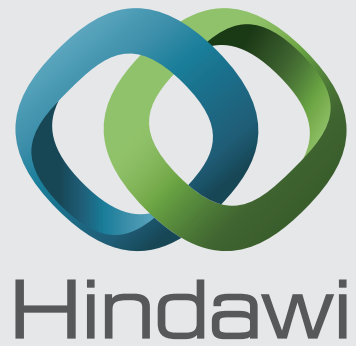

Submit your manuscripts at

http://www.hindawi.com
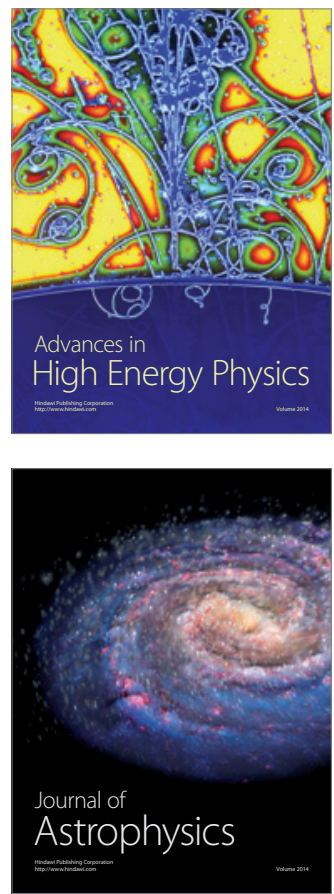
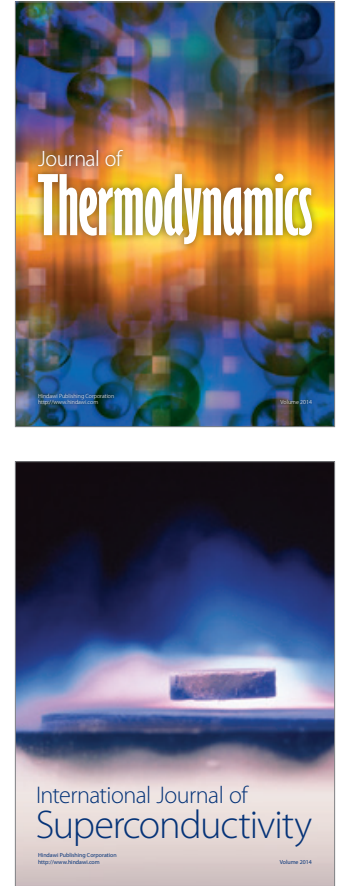
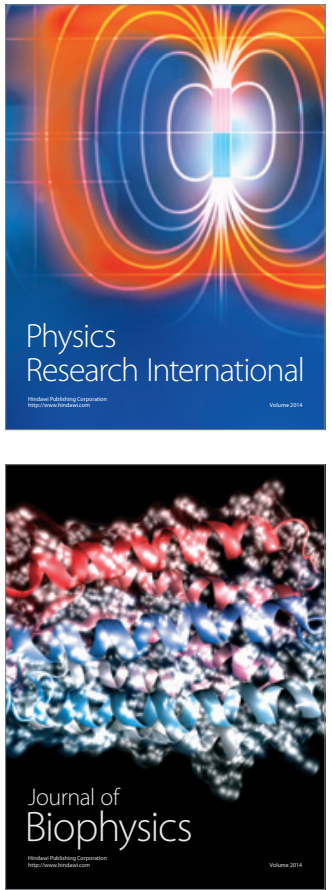
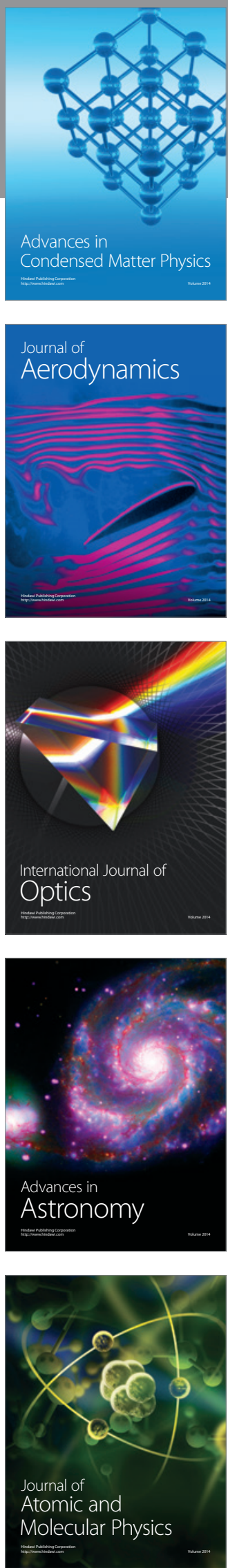